\title{
Mesenchymal stem cells-derived exosomal miRNA- 28-3p promotes apoptosis of pulmonary endothelial cells in pulmonary embolism
}

Hongyu Mao

Cangzhou Central Hospital

Lina Liu

Cangzhou Central Hospital

Yamin Hu ( $\sim$ huyamin091@163.com)

Cangzhou Central Hospital https://orcid.org/0000-0003-4299-7745

\section{Research}

Keywords: pulmonary embolism, apoptosis, exosome, miRNA-28-3p, API5, AMSCs

Posted Date: June 19th, 2020

DOI: https://doi.org/10.21203/rs.3.rs-36047/v1

License: (c) (i) This work is licensed under a Creative Commons Attribution 4.0 International License.

Read Full License 


\section{Abstract \\ Background}

Pulmonary embolism (PE) is a primary clinical manifestation of venous thromboembolism (VTE). It has been demonstrated that pulmonary endothelial cells (PECs) are apoptotic-resistance in PE. In this study, PECs were collected from PE patients and mouse models. Western blot, RT-PCR, flow cytometry, H\&E and TUNEL assay, confocal and TEM microscopy, and luciferase reporter assay were performed to determine the effects of miR-28-3p on PECs apoptosis and if exosomes can act as the shuttle to transport miR-28$3 p$ to PECs.

\section{Material and Methods}

The results revealed that apoptosis and miR-28-3p were downregulated in PECs of PE. The miR-28-3p mimics and inhibitor enhanced and further inhibited apoptosis in PECs, respectively.

\section{Results}

Both miR-28-3p-modified adipose tissue-derived mesenchymal stem cells (AMSCs) and AMSC-derived exosomes upregulated miR-28-3p expression in PECs, leading to elevated apoptosis of PECs. Apoptosis inhibitor 5 (API5) was a direct target gene of miR-28-3p, and the overexpression of API5 in miR-28-3pmodified PECs further suppressed apoptosis.

\section{Conclusions}

Furthermore, the administration of miR-28-3p-modified exosomes to PE mouse model promoted apoptosis in PECs. In conclusion, exosomal miR-28-3p could ameliorate PE-associated apoptosisresistance in PECs through targeting API5 in vitro and in vivo. Therefore, AMSCs-derived exosome is a promising way to deliver functioning miRNA to PECs, providing insight into novel therapy of PE.

\section{Introduction}

Pulmonary embolism (PE) is a common and deadly venous thromboembolic disease, which is the thirdleading cause of cardiovascular death [1]. The outcomes of PE widely range from asymptomatic, accidental emboli to a lethal massive embolism [2]. It has been reported that clinically apparent and untreated PT is related to about thirty percent of hospital mortality, while the mortality rate of treated PE patients substantially drops to about eight percent $[3,4]$. Despite all the research conducted in the past decades, including prophylaxis, diagnostic standards, and therapeutic strategies, PE is still regarded as a commonly underdiagnosed and highly lethal disorder [5]. Thus, further exploration of the mechanism of PE would be beneficial to both basic and clinical research of PE. 
MicroRNAs (miRNAs), 19-25 nucleotides in length, are a class of small noncoding RNA molecules that exert roles in post-transcriptional regulation to various biological and pathological processes [6]. In particular, miRNAs bind 3'UTR of mRNAs, thereby silencing gene expression through directly inhibiting translation or promoting mRNA degradation [7]. To date, several studies demonstrate that miRNAs are promising biomarkers for the diagnosis of PE including miRNA-134 [7], miR-28-3p [8], miR-27a/b [9], miR221 [10], and miRNA-1233 [11], where they are all significantly up-regulated in patients with PE. However, as an important regulator of gene expression, little attention has been given to the function of miRNAs in the pathological progression of $\mathrm{PE}$.

Apoptosis is a classic process of programmed cell death that is highly associated with cell development and aging [12]. Apoptosis functions as a homeostatic modulator to regulate the dynamics of the cell population in the multicellular organism [12]. It has been demonstrated that apoptosis plays an important role in a variety of disorders, such as lung and kidney cancer [13, 14], cardiovascular disease [15], neurodegeneration [16], and hepatobiliary disease [17]. It is well-documented that PE is tightly associated with pulmonary hypertension $[2,18]$, leading to an aberrant balance of cell proliferation and apoptosis in which cells, such as pulmonary endothelial cells (PECs), are hyper-proliferated and apoptosis-resistant $[19,20]$. However, the mechanism underlining PE-associated apoptosis-resistance is not fully understood.

Intercellular communication is one of the most important characteristics of multicellular organisms, of which extracellular vesicles (EVs), including exosomes, microvesicles, and prostasomes, have been identified in recent decades, although some of these vesicles are still poorly understood [21, 22]. Exosomes, one of the most popular EVs, are specialized nano-sized vesicles of endocytic origin and have been demonstrated to function as a vehicle for intercellular transfer of functioning molecules, such as RNA, lipids, and protein [23]. So far, reports suggest that exosomes are an ideal way to transport miRNA between cells in vitro $[24,25]$. Therefore, this study was aimed to determine the function of miR-28-3p in apoptosis-resistance of PECs in PE models and to see if adipose tissue-derived mesenchymal stem cells (AMSCs)-derived exosomes can function as an ideal vehicle to transport miR-28-3p to PECs.

\section{Materials And Methods}

\section{Ethics statement}

In this study, all subjects were informed before inclusion, and written consents were given by their guardians. All animal experiments were performed in accordance with the guidelines of the local regulatory agencies and conformed to the Regulations for the Management of Laboratory Animals published by the Ministry of Science and Technology of the People's Republic of China. All the experimental protocols were approved by the Institutional Animal Care and Use Committee of Cangzhou Central Hospital.

\section{Establishment of PE mouse model}


Twelve male C57BL/ 6 mice ( $20.1 \pm 1.87 \mathrm{~g}$, 8 weeks old; Jackson Labs, Bar Harbor, USA) were randomly divided into two groups ( $n=6$ per group): control (sham) and PE. The PE model was established by intravenous injection of the autologous thrombi which followed the detailed protocol from the previous study [26]. Two days post-injection, mice were sacrificed and the pulmonary arteries were collected.

\section{Primary PECs collection}

Thirteen patients with PE ( 6 men and 7 women; aged $39.5 \pm 6.7$ years) and 15 healthy subjects ( 9 men and 6 women; aged $37.2 \pm 5.1$ years) were enrolled in this study. The inclusion criterion of PE was followed by previous guidelines [27-29] (for example, systolic pulmonary artery pressure (sPAP) >= $40 \mathrm{~mm} \mathrm{Hg}$ ). Pulmonary artery samples were collected from patients with PE who underwent atrial septum treatment in humans or the PE procedure in mice. Human and mice PECs (hPECs and mPECs, respectively) were isolated from the pulmonary artery samples and cultured in an endothelial cellselective medium. The detailed protocols were described in previous studies [26, 28].

\section{AMSCs culture and transfection}

Human and mouse subcutaneous adipose tissues were obtained from the PE humans and the PE mice. Adipose tissues were harvested based on the protocol described in Liu et al., 2007 [30] and cultured in MesenPRO ${ }^{\circledR}$ RS $^{\text {TM }}$ Medium (1\% antibiotic-antimycotic; Gibco BRL, Gaithersburg, USA). AMSCs were confirmed by flow cytometric analysis of cell immunophenotype [31]. The tested surface markers of AMSCs included PE-labeled cluster designation 29 (CD29), CD31, CD44, CD45, CD73, CD 90, CD105, and human leukocyte antigen-DR (HLA-DR; BD Bioscience Pharmingen, San Diego, USA). MiR-28-3p was upregulated through transfection with lentivirus-mediated pre-miR-28-3p precursor (LV-miR-28-3p; Vigene Biosciences, Rockville, USA). LV-cel-miR-67 was used as the control. The transfection protocol was described in a previous study [31]. Isolated AMSCs were inoculated in a semi-permeable membrane (Transwell insert) in the upper part of 6-well culture plates, and PECs were inoculated in the lower part to establish a double-cell co-culture system [32]. After co-culture for 48 hours, the cells were harvested for subsequent assays.

\section{Transmission electron microscopy}

The AMSC-derived exosomes morphology was evaluated by transmission electron microscopy (TEM). Briefly, exosomes were fixed with $2 \%$ paraformaldehyde (PFA) and $3 \%(\mathrm{w} / \mathrm{v})$ glutaraldehyde in cacodylate buffer and then loaded to copper grids coated with Formvar. After washing, grids were contrasted with an equal amount of $10 \%$ uranyl acetate, dried, and then imaged by Morgagni 268D TEM with Advanced Microscopy Techniques digital imaging system (Advanced Microscopy Techniques Corp., Woburn, USA).

\section{Confocal microscopic assay}

Phospholipid membrane dye lipophilic carbocyanine DilC $_{16}$ (3) $(1.25 \mu \mathrm{M})$ was applied to labeled AMSCs) [33]. The protocol was described in a previous study [34]. PECs were cocultured with fluorescent exosomes originated from labeled AMSCs for 48 hours. Afterward, PECs were imaged by confocal microscopy (Olympus, Center Valley, USA). 


\section{PECs transfection}

MiR-28-3p mimics and inhibitor were purchased from GenePharma (GenePharma Co., Ltd, Shanghai, China). The transfection of miR-323-3p mimics and inhibitor into PECs were conducted according to the manufacturer's instructions of Lipofectamine ${ }^{T M} 3000$ Transfection Reagent (Invitrogen, Waltham, USA).

\section{Isolation of AMSC-derived exosome}

Exosomes were isolated from the supernatant of miR-28-3p-modified AMSCs by using ExoQuick-TC Kit (System Biosciences, CA, USA) according to the manufacturer's instructions. These exosomes were subsequently confirmed by western blotting analysis of the surface markers (CD9 and CD63; BD Bioscience Pharmingen, San Diego, USA). The morphology of exosomes was observed by Transmission Electron Microscopy (TEM). After centrifugal filtering, precipitates from the AMSCs-cultured medium were used as negative control. Isolated exosomes were co-cultured with PECs for 48 hours and then used in subsequent assays.

\section{Real-time PCR}

Total RNA was isolated from AMSCs, exosomes, and PECs using the Trizol Reagent kit (Sigma, St. Louis, USA) in accordance with the manufacturer's instructions. Reverse transcription of miRNA was performed by using stem-loop primers in TaqMan ${ }^{\text {TM }}$ Pri-miRNA Assay (AssayID: Hs03302668_pri; Thermo Fisher Scientific, MA, USA). The miRNA expressions were measured using the Fast SYBR Green Master Mix (Applied Biosystems, Carlsbad, USA) and the 7500 Fast Real-Time PCR System (Applied Biosystems, Carlsbad, USA). Real-time PCR data were analyzed using the comparative threshold cycle relativequantification method $\left(2^{-\Delta \Delta C t}\right)$, and the miRNA expression was normalized to that of U6 snRNA (housekeeping miRNA) as a reference control.

\section{Flow cytometry}

For the apoptosis assay, the cells were harvested and collected. Cell apoptosis was assessed using a FITC-Annexin V and PI kit (Invitrogen, Molecular Probes). The collected cells were suspended in $100 \mu \mathrm{L}$ of staining buffer, incubated for $15 \mathrm{~min}$, and kept on ice until analysis through flow cytometry. Cells, which were negative for both PI and FITC-Annexin V stainings were considered to be living cells, whereas cells that were PI-negative and FITC-Annexin V-positive were considered to be early apoptotic cells, and that showed both PI-positive and Annexin V-positive were those in the later stages of apoptosis [35]. The results were analyzed using the ModFit software and FACSCaliber (BD Bioscience, CA, USA).

\section{Luciferase reporter assay}

A sequence carrying the predicted binding sites of miR-28-3p was designed from the 3'UTR of API5 and then was inserted into the firefly luciferase reporter gene in PMIR (Ambion, Austin, USA). The accuracy of insertion was confirmed by sequencing. In addition, a sequence carrying mutated miR-28-3p binding sites was inserted into the same luciferase reporter to test binding specificity. The modified luciferase reporter plasmids carrying either API5-wide-type or API5-mutant were transfected with miR-28-3p or miR-control 
into PECs by using Lipofectamine ${ }^{\mathrm{TM}} 3000$ kit (Invitrogen, CA, USA) in accordance with the manufacturer's instructions. Twenty-four hours post-transfection, the luciferase assay kit (Promega, Madison, WI, USA) was applied to evaluate the luciferase activity.

\section{Western blot}

Total proteins were extracted from AMSCs and PECs by using RIPA lysis buffer (Beyotime Institute of Biotechnology, Shanghai, China). The detailed protocol was performed as previously described [36]. The primary antibodies for apoptosis inhibitor 5 (API5), CD9 and CD63, and GAPDH were purchased from Cell Signaling Technology (Cell Signaling Technology, MA, USA). Quantification of protein abundance was determined using the Uvitec Alliance software (Eppendorf, Hamburg, Germany).

\section{Plasmid construction}

To construct API5 overexpression plasmids, API5 cDNA was synthesized and inserted into a pLVX-cir vector (GenePharma Co., Ltd Shanghai, China) in accordance with the manufacturer's instructions. The empty plasmids were used as a negative control. Transfection was performed by using Lipofectamine 3000 (Invitrogen, Waltham, USA) in accordance with the manufacturer's instructions.

\section{Animals and experimental protocols}

Eighteen male C57BL/ 6 mice ( $21.2 \pm 1.51 \mathrm{~g}$, 8 weeks old; Jackson Labs, Bar Harbor, USA) were firstly used to establish the PE mouse model based on the protocol described above. The PE mice were randomly divided into three groups for different treatment: control, miR-67 modified exosome (exosome67), and miR-28-3p modified exosome (exosome-28-3p). Exosome pellets were resuspended in PBS solution at $5 \mu \mathrm{g} / \mu \mathrm{L}$ protein concentration and intravenously injected into mice ( $5 \mu \mathrm{g} / \mathrm{mouse})$. Three days post-injection, mice were sacrificed, and the pulmonary arteries were collected for subsequent assays.

\section{TUNEL assay}

Mouse arteries tissues were collected and fixed in 4\% paraformaldehyde in PBS containing $0.12 \mathrm{mM}$ sucrose for 15 minutes. Paraffin sections were made and subjected to TUNEL staining with the in situ TUNEL apoptosis detection kit (Beyotime Institute of Biotechnology, Nantong, China) according to the manufacturer's instructions. Sections were counterstained with 4',6-diamidino-2-phenylindole (DAPI), and the apoptotic cells were evaluated by fluorescence microscopy (Carl Zeiss, Meditec, Germany).

\section{Hematoxylin and eosin (H\&E) staining}

H\&E staining was performed for histopathological examination for lung tissues. Briefly, tissues were fixed in $10 \%(\mathrm{w} / \mathrm{v})$ neutral-buffered formalin for 24 hours, dehydrated with a graded ethanol series, and then embedded in paraffin. The lung tissue sections ( $5 \mu \mathrm{m}$ thickness) were mounted on gelatin-coated slides and stained with routine hematoxylin and eosin. After staining, the slides were imaged by using a light microscope (AmScope, Irvine, USA).

\section{Statistical analysis}


In this study, data were presented as means \pm SEM from at least four independent replicates. The statistical analyses were completed by using SPSS 13.0 software (SPSS, Chicago, USA). The differences between groups were calculated using one-way ANOVA or two-tailed Student's t-test. $P<0.05$ was considered statistically significant.

\section{Results}

\section{MiR-28-3p affected PECs apoptosis in PE}

Since miR-28-3p may be a diagnostic marker for PE due to its upregulation in the plasma [8], the expression of miR-28-3p was first detected in PECs from PE patients and mice. On the contrary, miR-28-3p was decreased in PECs of PE patients and mice (Figure. 1A and 1C). To test the effect of miR-28-3p on PECs apoptosis, the miR-28-3p mimics and inhibitor were applied to PECs of PE. Compared with the healthy controls, apoptosis levels were reduced in PECs of PE patients and mice (Figure. 1B and 1D). Also, the miR-28-3p mimics and inhibitor increased and further decreased the apoptosis of PECs, respectively. These results revealed that miR-28-3p plays an important role in regulating PECs apoptosis in PE.(Figure1)

\section{Construction of miR-28-3p-modified AMSCs}

o establish miR-28-3p-modified AMSCs, human and mouse AMSCs were transfected with LV-miR-28-3p. Real-time PCR results revealed that the expression of miR-28-3p significantly increased in miR-28-3ptreated AMCSs (AMCS-28-3p) compared with miR-67-treated AMCSs (AMSC-67) and control AMCSs (Figure. 2A and 2B). To confirm the cellular property of miR-28-3p-treated AMCSs, a flow cytometric assay was performed to detect the surface markers of engineered AMCSs. The results suggested that miR-283p-treated AMCSs were positive for CD29, CD44, CD73, CD90, and CD105, and negative for HLA-DR CD31 and CD45 in both hAMCSs and mAMCSs (Figure. 2C).(Figure2)

\section{MiR-28-3p-modified AMSCs promoted PECs apoptosis in PE}

To test the effect of miR-28-3p-modified AMSCs on PECs apoptosis in PE, PECs from PE patients and mice were cocultured with miR-28-3p-modified AMSCs for 48 hours. The miR-28-3p expression was significantly higher in PECs cocultured with miR-28-3p-modified AMSCs than those cocultured with the control AMSCs or miR-67-modified AMSCs (Figure. 3A and 3C). Furthermore, the apoptosis levels of PECs were enhanced in PECs treated with miR-28-3p-modified AMSCs in both humans and mice (Figure. 3B and 3D). These results further demonstrate the essential effect of miR-28-3p in PECs apoptosis and provide a clue that there may be an intercellular communication between AMSCs and PECs to mediate effects of miR-28-3p. (Figure3)

\section{Construction of miR-28-3p-modified exosomes}


It is well-documented that exosomes play important roles in intercellular communication, such as the delivery of functioning miRNAs [37-39]. Thus, exosomes and the supernatant were collected from miR28-3p-modified AMSCs. The property of exosome was confirmed by detecting exosome markers. Exosomes from miR-28-3p-modified AMSCs positively expressed CD9 and CD6 (Figure. 4A). TEM examination revealed the presence of AMSCs and exosomes that displayed a typical cup-shape (Figure. 4B). The exosome size distribution profile showed that the size of the majority of exosome $(98.6 \%)$ was around $134.0 \mathrm{~nm}$ (Figure. 4C). Also, the real-time PCR results showed that miR-28-3p expression was significantly higher in exosomes originated from miR-28-3p-modified AMSCs compared with those from miR-67-modified AMSCs and control AMSCs (Figure. 4E and 4F). To determine if exosomes derived from miR-28-3p-modified AMSCs mediated miR-28-3p-deliver, miR-28-3p-modified AMSCs were labeled with lipophilic carbocyanine DilC $_{16}(3)$. The exosomes from labeled AMSCs were then collected and cocultured with PECs. Confocal images revealed that the fluorescent dyes were detected in the membrane and cytoplasm of PECs without labeling, suggesting that AMSCs-derived exosomes delivered miR-28-3p from AMSCs into PECs (Figure. 4D). (Figure4)

\section{MiR-28-3p affected PECs apoptosis by targeting API5}

MiRNAs are regarded as essential regulators for gene expression [40]. To further determine the effect of miR-28-3p on PECs apoptosis, online bioinformatics tools, such as PicTar (http://pictar.mdc-berlin.de/), Miranda (http://microRNA.org) and TargetScan (www.targetscan.org/index.html) were used to predict potential target genes of miR-28-3P. Based on the predictions and the effect of miR-28-3p on PECs apoptosis, API5, an anti-apoptotic factor, was selected as a presumed target gene of miR-28-3P (Figure. $5 D)$. Protein and mRNA expression of API5 were decreased in PECs treated with miR-28-3P-modified exosomes (Figure. 5A, 5B, and 5C). The luciferase reporter assay revealed that transfection of miR-28-3p significantly decreased luciferase activity in PECs carrying the wide-type API5 3'UTR, whereas miR-28-3p did not affect the luciferase activity in PECs carrying the mutant API5 3 'UTR (Figure. 5E). These results suggested that API5 is a direct target gene of miR-28-3p. Next, to confirm the function of API5 in miR-28$3 p$-associated with PECs apoptosis, API5 overexpression was induced in PECs treated with miR-28-3Pmodified exosomes. In both human and mouse PECs, the overexpression of API5 significantly decreased the apoptosis level compared with PECs only treated with miR-28-3P-modified exosomes (Figure. 5F and $5 G)$. Collectively, it is suggested that the effect of miR-28-3p on PECs apoptosis is mediated by API5.

(Figure5)

\section{MiR-28-3p affected PECs apoptosis in vivo}

To determine if miR-28-3p could affect PECs apoptosis in vivo, miR-28-3P-modified exosomes were injected into PE mice. Three days post-injection, the expression of miR-28-3p in PECs of mice treated with miR-28-3P-modified exosomes was higher than those treated with miR-67-modified or control exosomes (Figure. 6A). Also, like the observation found in the in vitro studies, protein expression of API5 was decreased in PECs treated with miR-28-3P-modified exosomes compared with the other two control groups (Figure. 6B). Furthermore, the TUNEL assay revealed that the percentage of TUNEL-positive cells 
was higher in PECs of mice treated with miR-28-3P-modified exosomes (Figure. 6C). Meanwhile, H\&E staining showed that miR-28-3P-modified exosomes could attenuate pathological representation of PE mice, displaying less alveolar exudation and hemorrhage (Figure. 6D). Together, the results suggest that miR-28-3p plays an important role in the regulation of PECs apoptosis in vivo and that the exosome is a promising way to deliver functioning miRNA to PECs in vivo. (Figure6)

\section{Discussion}

In this study, miR-28-3p was downregulated in PECs from PE patients and mice, which is opposite to the observation from the plasma of patients with PE [8]. Also, PECs were found to be highly apoptosisresistant in PE models, which was reversed by upregulation of miR-28-3p through coculturing with miR28-3p-modified AMSCs and exosomes. Furthermore, APAF1 was the target gene of miR-28-3p, and APAF1/caspase-9 signaling mediated the effects of miR-28-3p on PECs apoptosis. Finally, the effect of exosomal miR-29-3p on PECs was also confirmed in vivo.

For either a single or recurrent episode, $\mathrm{PE}$ is thought to lead to pulmonary vascular obliteration that is sufficiently severe to cause pulmonary hypertension [41]. It has been reported that severe pulmonary hypertension results in enhanced proliferative potential of endothelial cells (ECs), fibroblasts, and smooth muscle cells [20]. Also, it is suggested that severe pulmonary arterial hypertension is associated with cancer-like cell futures, such as resistance to antigrowth signals, enhanced proliferation, and evasion of apoptosis [42]. In the current study, the apoptosis level of PECs was downregulated in PECs of both PE patients and mice, which is in agreement with previous studies reporting PE-induced apoptosis-resistance in PECs [43] and pulmonary fibroblasts [44]. Endothelial cells exert vital roles in maintaining vascular integrity and regulating thrombosis, which is essential for vascular homeostasis [45]. The disturbed balance of PECs proliferation and apoptosis in PE may be associated with vascular remodeling since irregular thickening of the vessel is thought of as a main vascular sign of PE [46].

It has been demonstrated that miR-28-3p is a plasma diagnostic marker in PE [8], implying the potential roles of miR-28-3p in PE models. Thus, to further determine the underlying mechanism of PE, the focus was on the effect of miR-28-3p in PECs apoptosis-resistance in PE. In this study, the expression of miR28-3p was downregulated in PECs of both PE patients and the mouse model along with decreased PECs apoptosis, whereas the upregulation of miR-28-3p by engineered AMSCs and exosomes enhanced the level of apoptosis, indicating the potential roles of miR-28-3p in PECs apoptosis. As a multifunctional regulator, miR-28-3p is involved in various pathological processes. In ovarian cancer, miR-28-3p may play roles in cancer cell motility and invasion $[47,48]$. Furthermore, miR-28-3p is found to act as a cellular restriction regulator to suppress replication and infection of human T cell leukemia virus, type 1 [49]. Regarding apoptosis, upregulation of miR-28-3p regulates osteoblasts proliferation and apoptosis to promote fracture healing [50].

Notably, the expression of miR-28-3p detected in PECs disagrees with the report that the plasma level of miR-28-3p elevates in PE patients [8]. The similar paradoxical expression pattern of miRNAs between 
tissue and plasma has also been reported in prostate cancer [51]. Since the mechanism underlying releasing of miRNAs into the extracellular environment is still not completely clear, this phenomenon may be interpreted by several hypotheses, such as origination from damaged cells [52], microvesicles [39], protective proteins [53], and immunocytes [54]. However, the exact mechanism of this discrepancy should be further explored in the future.

In this study, API5 was identified as a direct target gene of miR-28-3p, and the overexpression of API5 could reverse the effect of the upregulation of miR-28-3p on PECs apoptosis, which indicates API5 signaling plays important roles in miR-28-3p-related PECs apoptosis in PE. Moreover, API5, also known as fibroblast growth factor-2-interacting factor (FIF) or anti-apoptosis clone 11 (AAC-11), was originally found to act as an anti-apoptotic factor in mouse fibroblasts [55], cervical carcinoma cells [56], and liver cells [57]. It is reported that API5 depletion enhances cellular sensitivity to the anti-tumor drug, indicating the anti-apoptotic role of API5 in cancer cell survival [58]. The anti-apoptotic effect of API5 displays an E2 promoter-binding factors (E2F)-dependent pattern [59]. The present study revealed that overexpression of API5 could downregulate apoptosis in exosome-treated PECs, indicating that miR-28-3p/API5 signaling may play important roles in regulating PECs apoptosis in PE.

As one of the important goals in this study, the function of AMSCs-derived exosomes as the vehicle to deliver miR-28-3p to PECs was investigated. The present study suggested that miR-28-3p was highly expressed in modified AMSCs and exosomes, and miR-28-3p expression was also upregulated in PECs after coculturing with exosomes, thereby alleviating apoptosis-resistance of PECs. Together, these results suggest that AMSC exosomes are operable and an efficient way to transport miRNA to PECs. As a promising approach, the specificity, accessibility, and stability of exosome-carried miRNAs make them a high-efficiency way to regulate cellular functions and activities [37], which is widely applied in multiple studies. For example, AMSC-originated exosomal miR-122 can enhance hepatocellular carcinoma chemosensitivity [60]. In addition, AMSC-derived exosomes elevate miR-17 expression in hepatic macrophages, thereby ameliorating acute liver failure in mice [61]. Therefore, AMSC exosome-based transportation of miRNAs may be a promising therapeutic strategy.

In conclusion, in this study, miR-28-3p was downregulated in PECs from both PE patients and mice, which is opposite to the observation from the plasma of patients with PE [8]. Also, PECs were found to be highly apoptosis-resistant in PE models, which could be reversed by the miR-28-3p upregulation through coculturing with miR-28-3p-modified AMSCs or exosomes. Furthermore, API5 was the target gene of miR28-3p, and API5 mediated the effects of miR-28-3p on PECs apoptosis. Finally, the effect of exosomal miR-29-3p on PECs was also confirmed in the PE mice model in vivo. The results suggested that miR-28$3 p$ could ameliorate PE-associated apoptosis-resistance in PECs through targeting API5, and AMSCsderived exosomes are an ideal way to deliver functional miRNA to PECs for PE treatment. Thus, this study may provide a novel strategy to treat PE disease.

\section{Conclusions}


In this study, data were presented as means \pm SEM from at least four independent replicates. The statistical analyses were completed by using SPSS 13.0 software (SPSS, Chicago, USA). The differences between groups were calculated using one-way ANOVA or two-tailed Student's t-test. $P<0.05$ was considered statistically significant.

\section{Abbreviations}

Pulmonary embolism (PE)

adipose tissue-derived mesenchymal stem cells (AMSCs)

Apoptosis inhibitor 5 (API5)

pulmonary endothelial cells (PECs)

venous thromboembolism (VTE)

\section{Declarations}

In this study, all subjects were informed before inclusion, and written consents were given by their guardians. All animal experiments were performed in accordance with the guidelines of the local regulatory agencies and conformed to the Regulations for the Management of Laboratory Animals published by the Ministry of Science and Technology of the People's Republic of China. All the experimental protocols were approved by the Institutional Animal Care and Use Committee of Cangzhou Central Hospital.

\section{Consent for publication}

None applicable

\section{Availability of data and materials}

The datasets used and/or analysed during the current study are available from the corresponding author on reasonable request.

\section{Competing interests}

The authors declare that they have no competing interests 


\section{Author Contributions:}

Hongyu Mao performed the majority of experiments and analyzed the data; Lina Liu performed the molecular investigations; Yamin Hu designed and coordinated the research; Yamin Hu wrote the paper.

\section{Funding:}

This study is funded by Natural Science Foundation of Hebei Province (Grant No. H0214).

\section{Conflicts of Interest:}

The author reports no conflicts of interest in this work.

\section{Acknowledge [}

None

\section{References}

1. Turetz M, Sideris AT, Friedman OA et al, editors. Epidemiology, pathophysiology, and natural history of pulmonary embolism. Seminars in interventional radiology; 2018: Thieme Medical Publishers.

2. Pengo $\mathrm{V}$, Lensing AW, Prins $\mathrm{MH}$, et al. Incidence of chronic thromboembolic pulmonary hypertension after pulmonary embolism. N Engl J Med. 2004;350(22):2257-64.

3. Dalen JE, Alpert JS. Natural history of pulmonary embolism. Prog Cardiovasc Dis. 1975;17(4):25970.

4. Hermann RE, Davis JH, Holden WD. Pulmonary embolism: a clinical and pathologic study with emphasis on the effect of prophylactic therapy with anticoagulants. The American Journal of Surgery. 1961;102(1):19-28.

5. Wood KE. Major pulmonary embolism: review of a pathophysiologic approach to the golden hour of hemodynamically significant pulmonary embolism. Chest. 2002;121(3):877-905.

6. Engels BM, Hutvagner G. Principles and effects of microRNA-mediated post-transcriptional gene regulation. Oncogene. 2006 Oct 9;25(46):6163-9.

7. Xiao J, Jing Z-C, Ellinor PT, et al. MicroRNA-134 as a potential plasma biomarker for the diagnosis of acute pulmonary embolism. Journal of translational medicine. 2011;9(1):159. 
8. Zhou X, Wen W, Shan X, et al. MiR-28-3p as a potential plasma marker in diagnosis of pulmonary embolism. Thrombosis research. 2016;138:91-5.

9. Wang Q, Ma J, Jiang Z, et al. Diagnostic value of circulating microRNA-27a/b in patients with acute pulmonary embolism. Int Angiol. 2018 Feb;37(1):19-25.

10. Liu T, Kang J, Liu F. Plasma Levels of microRNA-221 (miR-221) are Increased in Patients with Acute Pulmonary Embolism. Medical science monitor: international medical journal of experimental and clinical research. 2018 Nov 28;24:8621-8626.

11. Kessler T, Erdmann J, Vilne B, et al. Serum microRNA-1233 is a specific biomarker for diagnosing acute pulmonary embolism. Journal of translational medicine. 2016;14(1):120.

12. Elmore S. Apoptosis: a review of programmed cell death. Toxicol Pathol. 2007 Jun;35(4):495-516.

13. Pujals A, Renouf B, Robert A, et al. Treatment with a BH3 mimetic overcomes the resistance of latency III EBV (+) cells to p53-mediated apoptosis. Cell Death Dis. 2011 Jul;28(7):e184. 2(.

14. Gobé $\mathrm{G}$, Rubin $\mathrm{M}$, Williams $\mathrm{G}$, et al. Apoptosis and expression of $\mathrm{Bcl}-2, \mathrm{Bcl}-\mathrm{XL}$, and $\mathrm{Bax}$ in renal cell carcinomas. Cancer investigation. 2002;20(3):324-32.

15. Haunstetter A, Izumo S. Apoptosis: basic mechanisms and implications for cardiovascular disease. Circulation research. 1998;82(11):1111-29.

16. Bossy-Wetzel E, Barsoum MJ, Godzik A, et al. Mitochondrial fission in apoptosis, neurodegeneration and aging. Curr Opin Cell Biol. 2003;15(6):706-16.

17. Patel T, Gores GJ. Apoptosis and hepatobiliary disease. Hepatology. 1995;21(6):1725-41.

18. Becattini C, Agnelli G, Pesavento R, et al. Incidence of chronic thromboembolic pulmonary hypertension after a first episode of pulmonary embolism. Chest. 2006;130(1):172-5.

19. Masri FA, Xu W, Comhair SA, et al. Hyperproliferative apoptosis-resistant endothelial cells in idiopathic pulmonary arterial hypertension. Am J Physiol Lung Cell Mol Physiol. 2007 Sep;293(3):L548-54.

20. Wang L, Gan H-L, Liu Y, et al. The distinguishing cellular features of pulmonary artery smooth muscle cells from chronic thromboembolic pulmonary hypertension patients. Experimental lung research. 2013;39(8):349-58.

21. Raposo G, Stoorvogel W. Extracellular vesicles: exosomes, microvesicles, and friends. J Cell Biol. 2013;200(4):373-83.

22. Simons M, Raposo G. Exosomes-vesicular carriers for intercellular communication. Curr Opin Cell Biol. 2009;21(4):575-81.

23. Zomer A, Vendrig T, Hopmans ES, et al. Exosomes: Fit to deliver small RNA. Communicative \& integrative biology. 2010 Sep;3(5):447-50.

24. Zhang X, Yuan X, Shi H, et al. Exosomes in cancer: small particle, big player. J Hematol Oncol. 2015 Jul;10:8:83.

25. O'Loughlin AJ, Woffindale CA, Wood MJ. Exosomes and the emerging field of exosome-based gene therapy. Current gene therapy. 2012 Aug;12(4):262-74. 
26. Wan J, Lu L-J, Miao R, et al. Alterations of bone marrow-derived endothelial progenitor cells following acute pulmonary embolism in mice. Experimental Biology Medicine. 2010;235(8):989-98.

27. Konstantinides SV, Torbicki A, Agnelli G, et al. Guidelines on the diagnosis and management of acute pulmonary embolism: the Task Force for the Diagnosis and Management of Acute Pulmonary Embolism of the European Society of Cardiology (ESC). European heart journal. 2014;35(43):303373.

28. Li Y, Geng X, Wang H, et al. CTRP9 ameliorates pulmonary arterial hypertension through attenuating inflammation and improving endothelial cell survival and function. J Cardiovasc Pharmacol. 2016;67(5):394-401.

29. Chen Y, Wang C, Shang H, et al. Clinical practice guidelines in China. BMJ. 2018;360:j5158.

30. Liu Y, Yan X, Sun Z, et al. Flk-1 + adipose-derived mesenchymal stem cells differentiate into skeletal muscle satellite cells and ameliorate muscular dystrophy in mdx mice. Stem Cells Dev. 2007 Oct;16(5):695-706.

31. Lou G, Yang Y, Liu F, et al. MiR-122 modification enhances the therapeutic efficacy of adipose tissuederived mesenchymal stem cells against liver fibrosis. J Cell Mol Med. 2017 Nov;21(11):2963-73.

32. Su M-J, Aldawsari $\mathrm{H}$, Amiji M. Pancreatic cancer cell exosome-mediated macrophage reprogramming and the role of microRNAs 155 and 125b2 transfection using nanoparticle delivery systems. Scientific reports. 2016;6:30110.

33. Ismail $\mathrm{N}$, Wang $\mathrm{Y}$, Dakhlallah $\mathrm{D}$, et al. Macrophage microvesicles induce macrophage differentiation and miR-223 transfer. Blood. 2013;121(6):984-95.

34. Lou G, Yang Y, Liu F, et al. MiR-122 modification enhances the therapeutic efficacy of adipose tissuederived mesenchymal stem cells against liver fibrosis. J Cell Mol Med. 2017;21(11):2963-73.

35. Zhang FZ, Ho DH, Wong RH. Triptolide, a HSP90 middle domain inhibitor, induces apoptosis in triple manner. Oncotarget. 2018 Apr 27;9(32):22301-22315.

36. Pulito C, Mori F, Sacconi A, et al. Metformin-induced ablation of microRNA 21-5p releases Sestrin-1 and CAB39L antitumoral activities. Cell Discov. 2017;3:17022.

37. Zhao L, Liu W, Xiao J, et al. The role of exosomes and "exosomal shuttle microRNA" in tumorigenesis and drug resistance. Cancer letters. 2015;356(2):339-46.

38. Pegtel DM, Cosmopoulos K, Thorley-Lawson DA, et al. Functional delivery of viral miRNAs via exosomes. Proceedings of the National Academy of Sciences. 2010;107(14):6328-6333.

39. Valadi H, Ekström K, Bossios A, et al. Exosome-mediated transfer of mRNAs and microRNAs is a novel mechanism of genetic exchange between cells. Nat Cell Biol. 2007;9(6):654.

40. He L, Hannon GJ. MicroRNAs: small RNAs with a big role in gene regulation. Nat Rev Genet. 2004;5(7):522.

41. Hoeper MM, Mayer E, Simonneau, Gr, et al. Chronic thromboembolic pulmonary hypertension. Circulation. 2006;113(16):2011-20. 
42. Sakao S, Tatsumi K. Vascular remodeling in pulmonary arterial hypertension: multiple cancer-like pathways and possible treatment modalities. Int J Cardiol. 2011;147(1):4-12.

43. Masri FA, Xu W, Comhair SA, et al. Hyperproliferative apoptosis-resistant endothelial cells in idiopathic pulmonary arterial hypertension. American Journal of Physiology-Lung Cellular Molecular Physiology. 2007;293(3):L548-54.

44. Li S, Tabar SS, Malec V, et al. NOX4 regulates ROS levels under normoxic and hypoxic conditions, triggers proliferation, and inhibits apoptosis in pulmonary artery adventitial fibroblasts. Antioxid Redox Signal. 2008;10(10):1687-98.

45. Sage E, Mercier O, Van den Eyden F, et al. Endothelial cell apoptosis in chronically obstructed and reperfused pulmonary artery. Respiratory research. 2008;9(1):19.

46. Nishiyama KH, Saboo SS, Tanabe Y, et al. Chronic pulmonary embolism: diagnosis. Cardiovasc Diagn Ther. 2018;8(3):253-71.

47. Yeung CLA, Yeung T-L, Mok SC. Leptin facilitates ovarian cancer metastasis through miR-28-3p and matrix metalloproteinase-1 in the omental tumor microenvironment. AACR; 2018.

48. Báez-Vega PM, Vargas IME, Valiyeva F, et al. Targeting miR-21-3p inhibits proliferation and invasion of ovarian cancer cells. Oncotarget. 2016;7(24):36321.

49. Bai XT, Nicot C. miR-28-3p is a cellular restriction factor that inhibits human T cell leukemia virus, type 1 (HTLV-1) replication and virus infection. J Biol Chem. 2015;290(9):5381-90.

50. Xu D, Zhang G, Wu Y, et al. MicroRNA-28-3p promotes fracture healing through inhibition of Sox6 and activation of PI3K/AKT pathway. INTERNATIONAL JOURNAL OF CLINICAL AND EXPERIMENTAL PATHOLOGY. 2017;10(5):5263-5273.

51. Zedan AH, Hansen TF, Assenholt J, et al. microRNA expression in tumour tissue and plasma in patients with newly diagnosed metastatic prostate cancer. Tumor Biology. 2018;40(5):1010428318775864.

52. Turchinovich A, Weiz L, Langheinz A, et al. Characterization of extracellular circulating microRNA. Nucleic acids research. 2011;39(16):7223-33.

53. Wang K, Zhang S, Weber J, et al. Export of microRNAs and microRNA-protective protein by mammalian cells. Nucleic acids research. 2010;38(20):7248-59.

54. Merkerova M, Vasikova A, Belickova $\mathrm{M}$, et al. MicroRNA expression profiles in umbilical cord blood cell lineages. Stem Cells Dev. 2010;19(1):17-26.

55. Tewari $M, Y u M$, Ross $B$, et al. AAC-11, a novel cDNA that inhibits apoptosis after growth factor withdrawal. Cancer Res. 1997 Sep 15;57(18):4063-9.

56. Kim JW, Cho HS, Kim JH, et al. AAC-11 overexpression induces invasion and protects cervical cancer cells from apoptosis. Laboratory investigation. 2000;80(4):587.

57. Wang Y, Lee AT, Ma JZ, et al. Profiling microRNA expression in hepatocellular carcinoma reveals microRNA-224 up-regulation and apoptosis inhibitor-5 as a microRNA-224-specific target. J Biol Chem. 2008;283(19):13205-15. 
58. Rigou P, Piddubnyak V, Faye A, et al. The antiapoptotic protein AAC-11 interacts with and regulates Acinus-mediated DNA fragmentation. EMBO J. 2009;28(11):1576-88.

59. Morris EJ, Michaud WA, Ji JY, et al. Functional identification of Api5 as a suppressor of E2Fdependent apoptosis in vivo. PLoS Genet. 2006 Nov;17(11):e196. 2(.

60. Lou G, Song X, Yang F, et al. Exosomes derived from miR-122-modified adipose tissue-derived MSCs increase chemosensitivity of hepatocellular carcinoma. J Hematol Oncol. 2015;8(1):122.

61. Liu Y, Lou G, Li A, et al. AMSC-derived exosomes alleviate lipopolysaccharide/d-galactosamineinduced acute liver failure by miR-17-mediated reduction of TXNIP/NLRP3 inflammasome activation in macrophages. EBioMedicine. 2018;36:140-50.

\section{Figures}

A

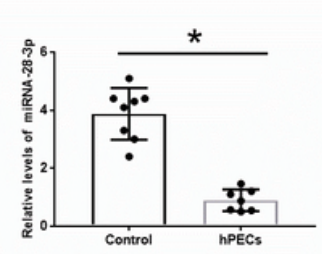

C

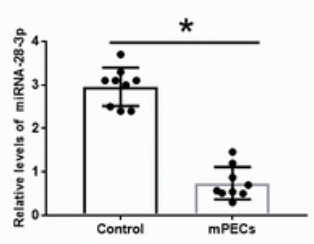

B

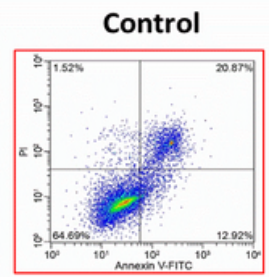

D

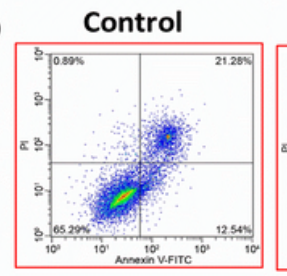

hPECS

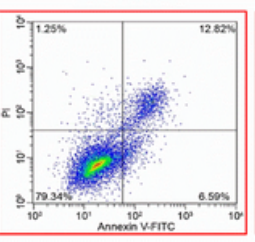

MPECS

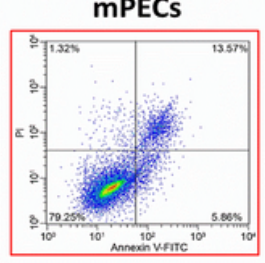

hPECs+mimics

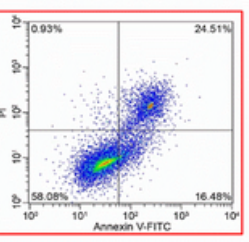

mPECs+mimics

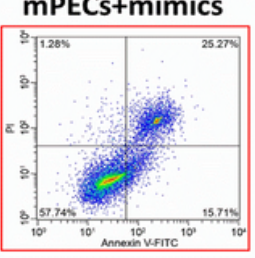

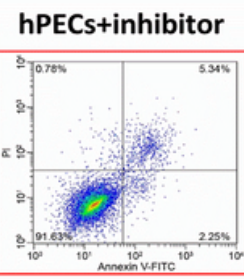

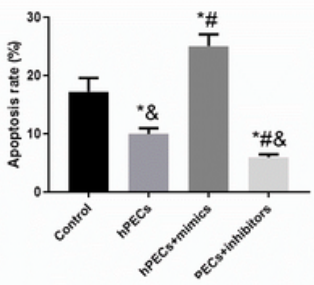

mPECs+inhibitor
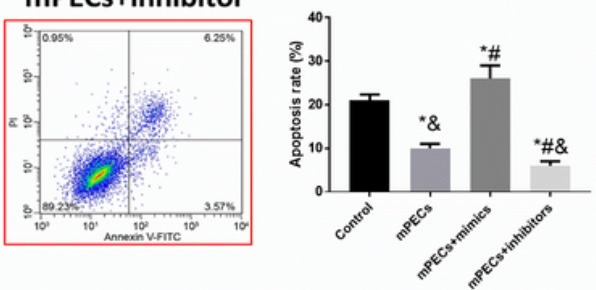

Figure 1

MiR-28-3p was downregulated in PECs. (A) miR-28-3p expression in hPECs. (B) After treating with miR-28$3 p$ mimics and inhibitor, hPECs apoptosis was analyzed by flow cytometry assay. (C) miR-28-3p expression in mPECs. (B) After treating with miR-28-3p mimics and inhibitor, mPECs apoptosis was analyzed by flow cytometry assay. Values are means \pm SEM. $* \mathrm{P}<0.05$ compared to control. \#P $<0.05$ compared to $\mathrm{hPECs} / \mathrm{mPECs}$. \&P $<0.05$ compared to $12 \mathrm{~h}$ exposure $\mathrm{hPECs} / \mathrm{mPECs}$ mimics. 

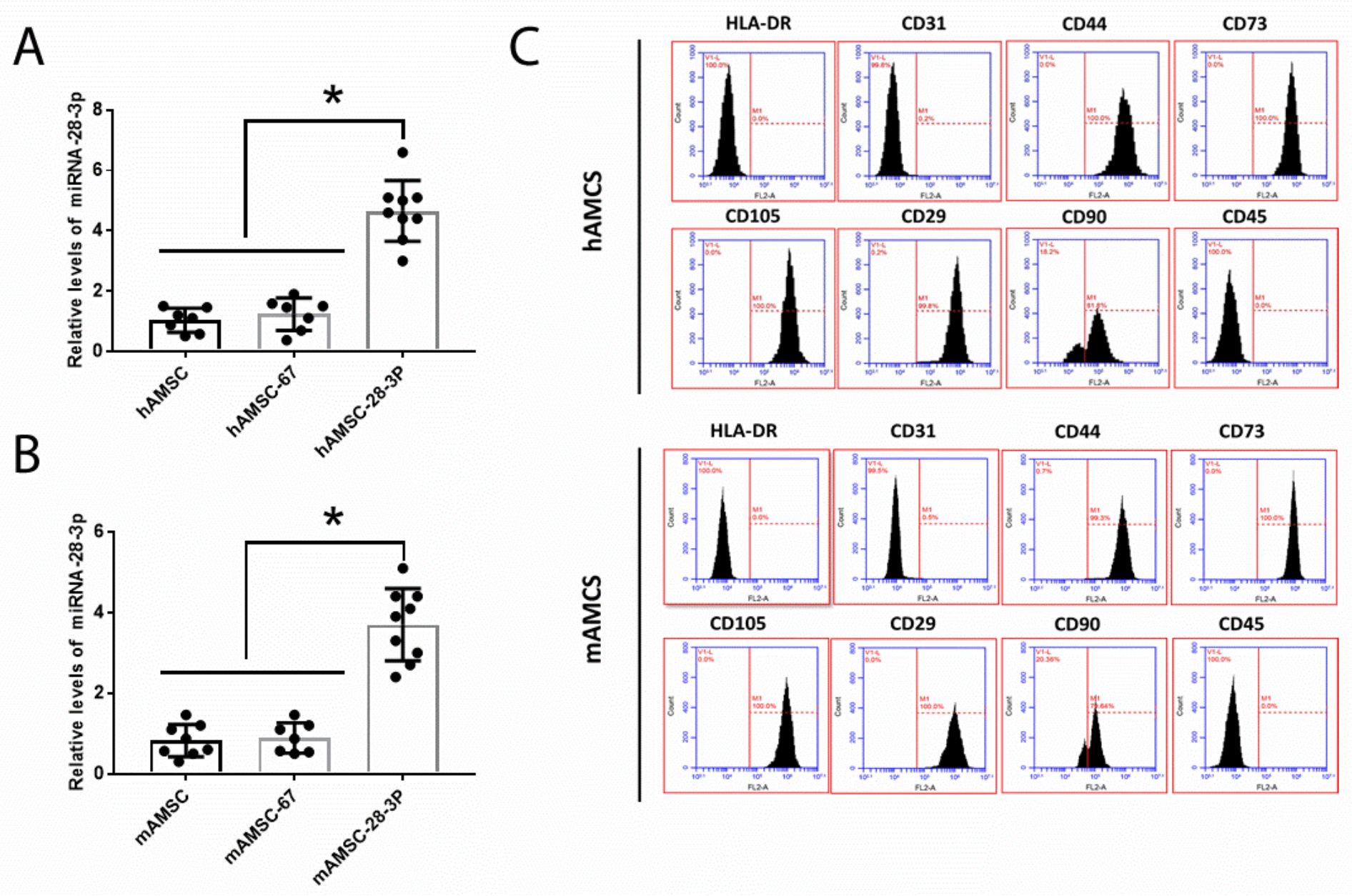

Figure 2

Isolation and confirmation of miR-28-3p-modified AMSCs. (A) miR-28-3p expression in hAMSCs. (B) miR28-3p expression in mAMSCs. (C) The surface markers of miR-28-3p-modified hAMSCs and mAMSCs were analyzed by flow cytometry assay. Values are means \pm SEM. $\left(^{*}\right)$ denotes the difference between groups $(P<0.05)$.

A

C
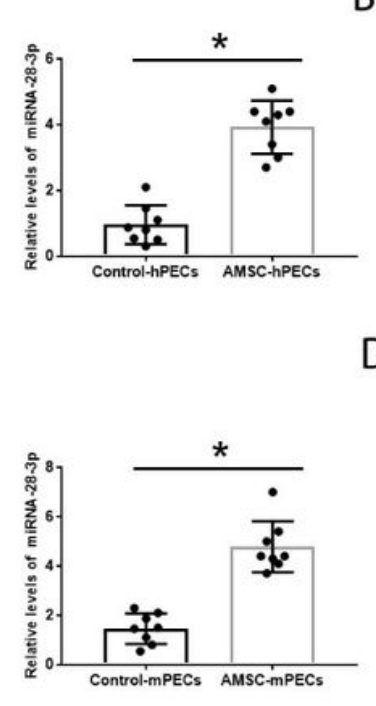

B

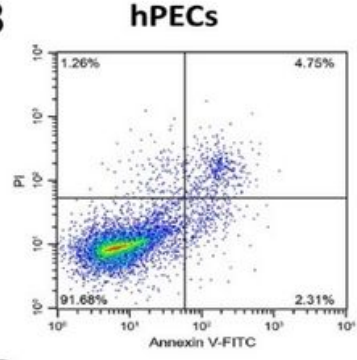

D

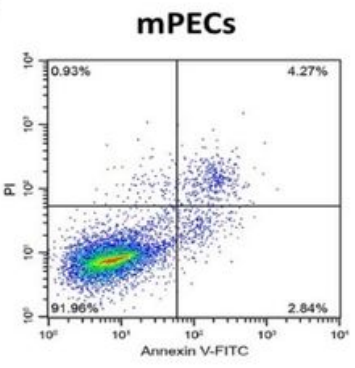

hPECS+AMSC-67

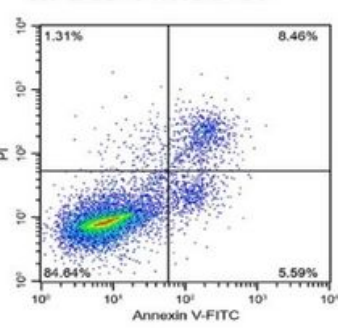

MPECS+AMSC-67

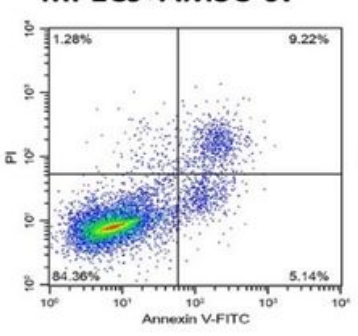

hPECS+AMSC-28-3p

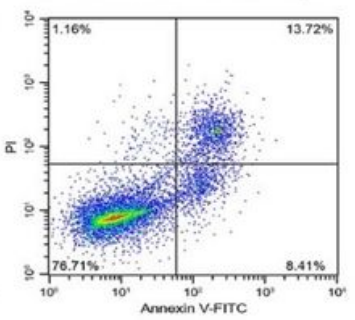

MPECS+AMSC-28-3p

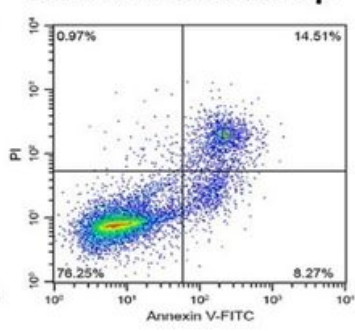

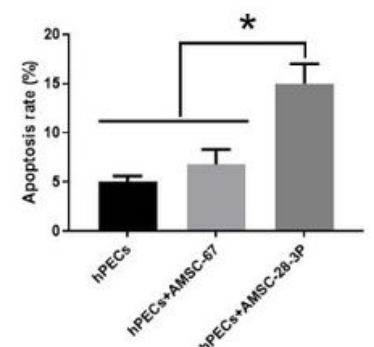

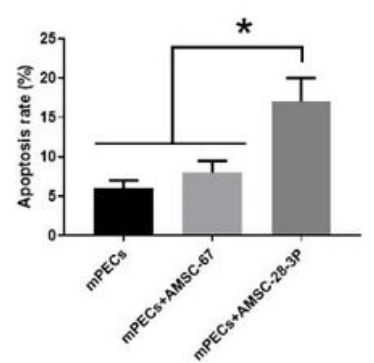




\section{Figure 3}

miR-28-3p-modified AMSCs affected PECs apoptosis. (A) miR-28-3p expression in hAMSCs-treated hPECs. (B) After treating with miR-28-3p-modified hAMSC, hPECs apoptosis was analyzed by flow cytometry assay. (C) miR-28-3p expression in mAMSCs-treated mPECs. (D) After treating with miR-28-3pmodified mAMSC, mPECs apoptosis was analyzed by flow cytometry assay. Values are means \pm SEM. ( ${ }^{*}$ ) denotes the difference between groups $(P<0.05)$.

A

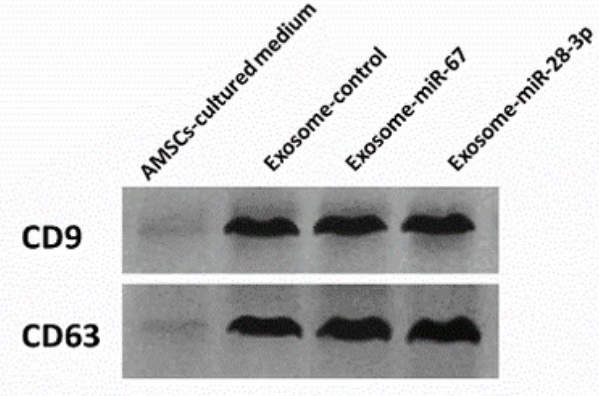

C

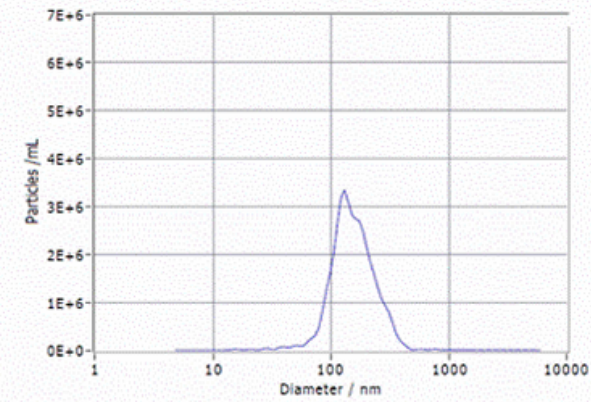

$\mathrm{E}$

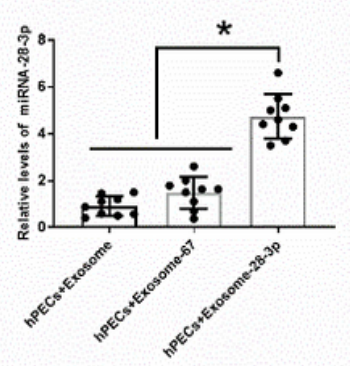

$\mathrm{F}$

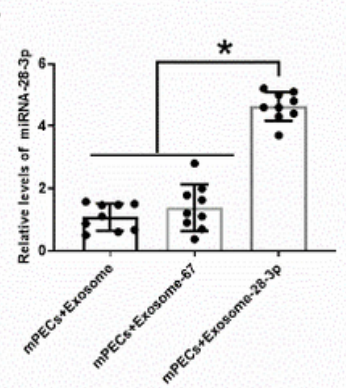

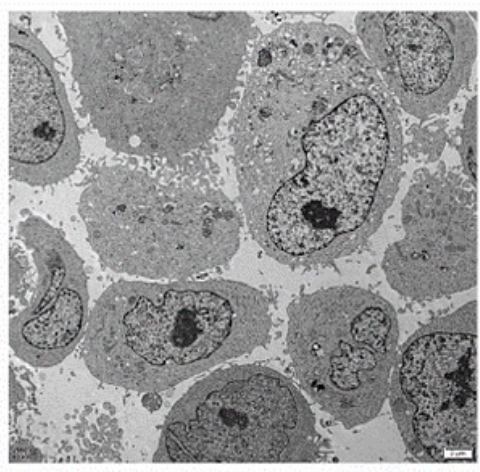

AMSCS

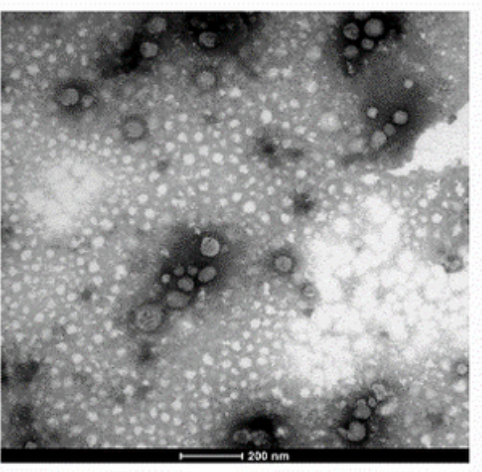

AMSC-derived exosomes
D

$$
\text { Dilc }_{16}(3)
$$

mAMSC-28-3p

$$
\text { Dilc }_{16}(3)
$$

MPECS

Exosome-28-3p

\section{Merge}

Merge

\section{Figure 4}

Isolation and confirmation of miR-28-3p-modified exosomes. (A) The surface markers of miR-28-3pmodified exosomes were analyzed by Western blot. (B) Transmission electron photomicrograph of AMSCs (left, scale bar $=2 \mu \mathrm{m}$ ) and AMSC-derived exosomes (right, scale bar $=200 \mathrm{~nm}$ ). (C) exosome size distribution. (D) Confocal images of miR-28-3p-modified mAMSC and exosome-28-3p-treated PECs $(\times 400)$. (E) miR-28-3p expression in hPECs treated with miR-28-3p-modified exosome. (F) miR-28-3p expression in $\mathrm{mPEC}$ s treated with miR-28-3p-modified exosome. Values are means $\pm \mathrm{SEM}$. ${ }^{*}$ ) denotes the difference between groups $(P<0.05)$. 
A

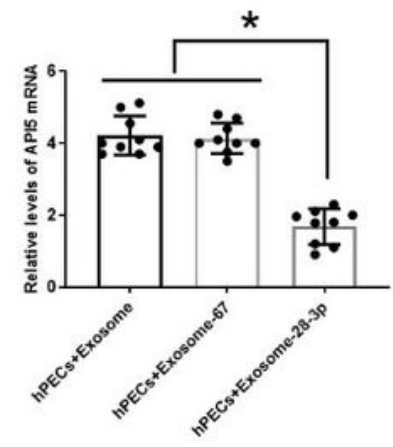

B

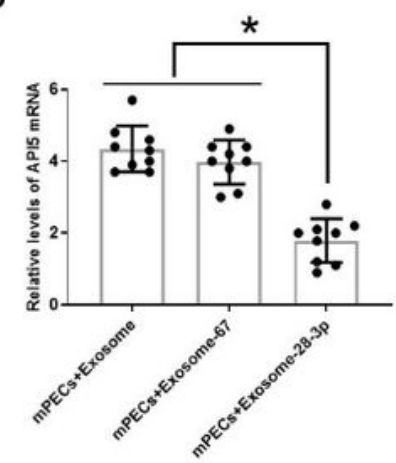

C

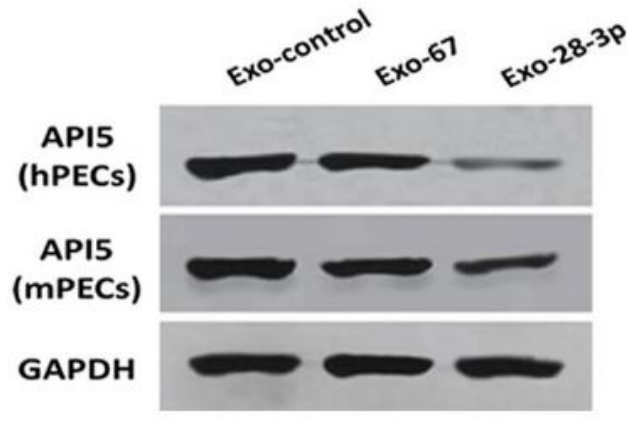

E

API5: 5' gugccauaucuggacUCGGCUu 3' (Mutant) II I

API5: 5 ' ugccugaaugacauuUCUAGUa 3' (Wide-type) IIIIII

miR-28-3p: 3' agguccucgaguguuAGAUCAC 5'

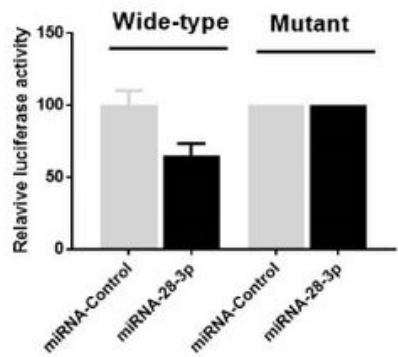

$\mathrm{F}$

hPECS

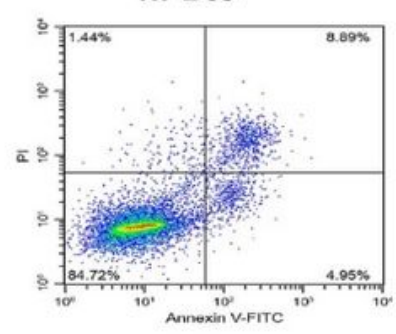

G

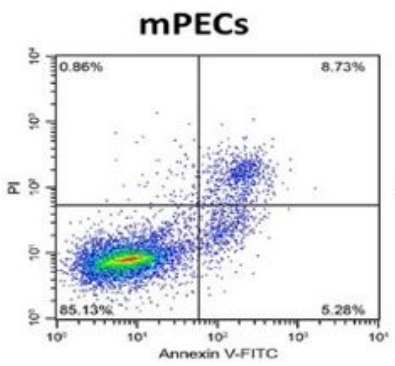

hPECS+Exo-28-3p

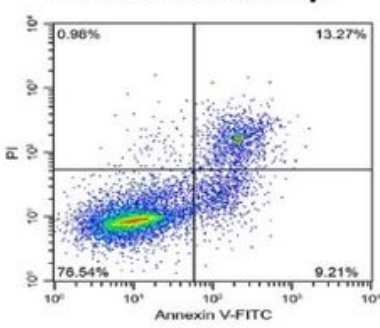

MPECS+Exo-28-3p

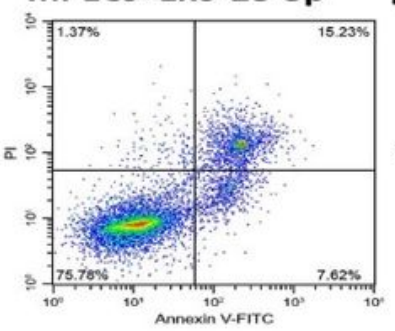

hPECs+Exo-28-3p+API5
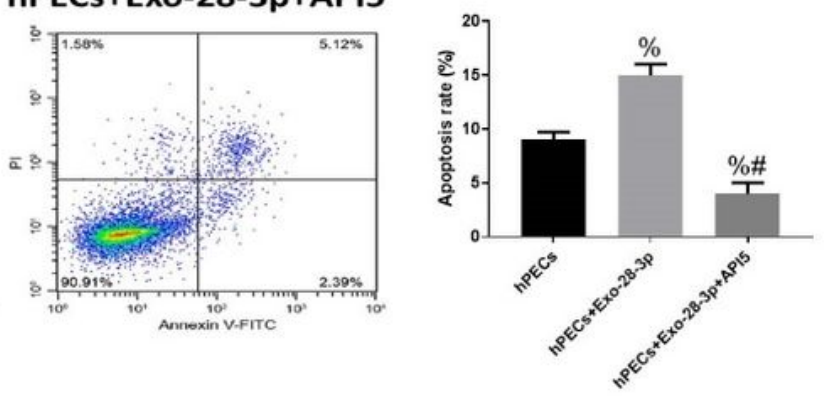

MPECs+Exo-28-3p+API5
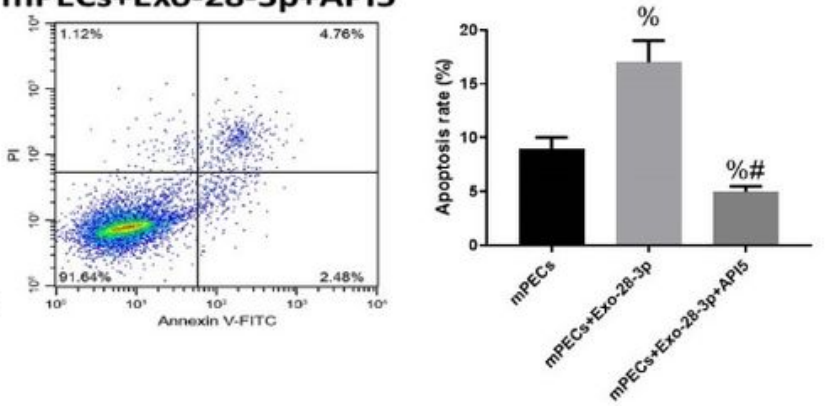

\section{Figure 5}

miR-28-3p affected PECs apoptosis by targeting API5. (A) API5 mRNA expression in hPECs treated with miR-28-3p-modified exosome. (B) API5 mRNA expression in mPECs treated with miR-28-3p-modified exosome. (C) API5 protein expression in hPECs and mPECs treated with miR-28-3p-modified exosome. (D) The sequence of wild-type and mutant miR-28-3p binding sites in the 3'UTR of API5. (E) Relative luciferase activity. (F) After overexpression of API5 in hPECs treated with miR-28-3p-modified exosome, 
hPECs apoptosis was analyzed by flow cytometry assay. (G) After overexpression of API5 in mPECs treated with miR-28-3p-modified exosome, mPECs apoptosis was analyzed by flow cytometry assay. Values are means \pm SEM. $\left.{ }^{*}\right)$ denotes difference between groups $(\mathrm{P}<.05) . \% \mathrm{P}<0.05$ compared to hPECs/mPECs. \#P< 0.05 compared to hPECs/mPECs+Exo-28-3p.

A

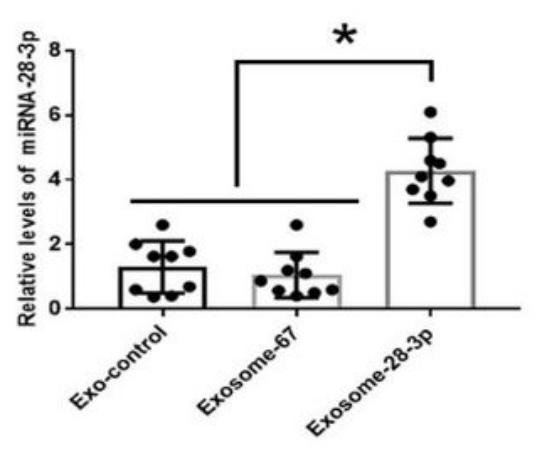

C

DAPI

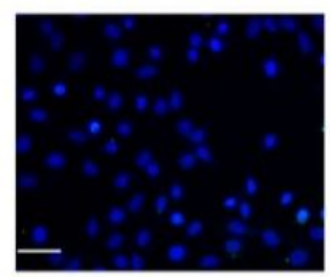

Exosome-28-3p
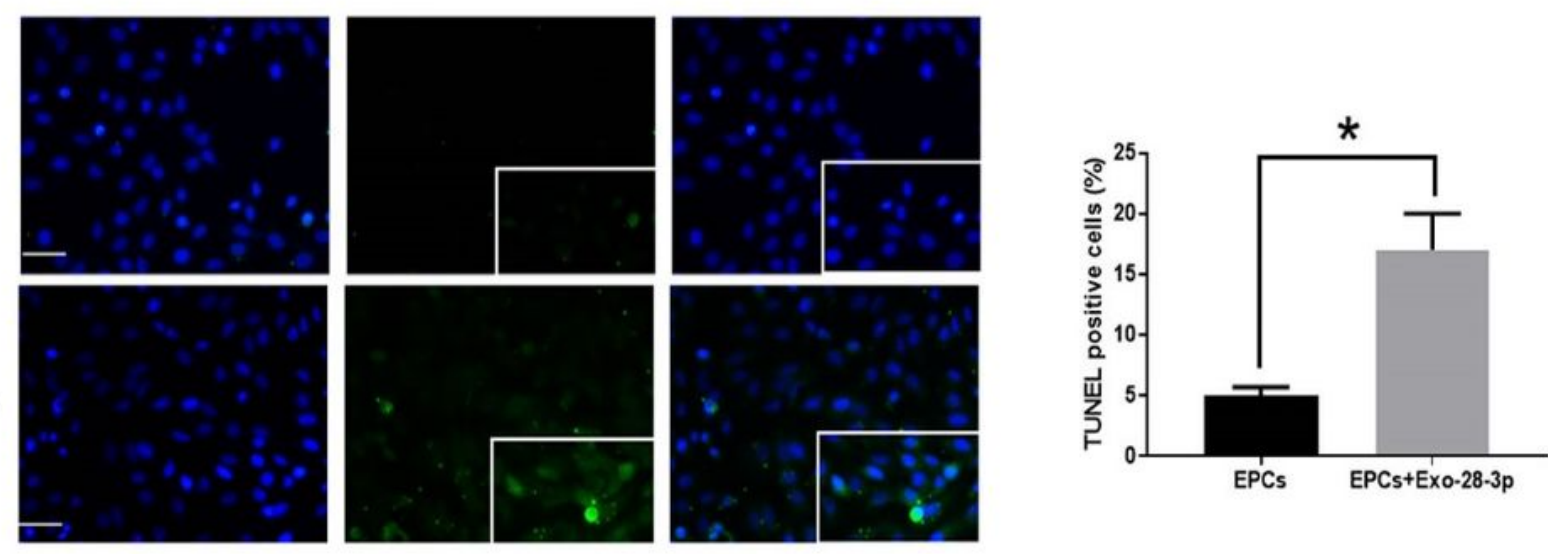

D

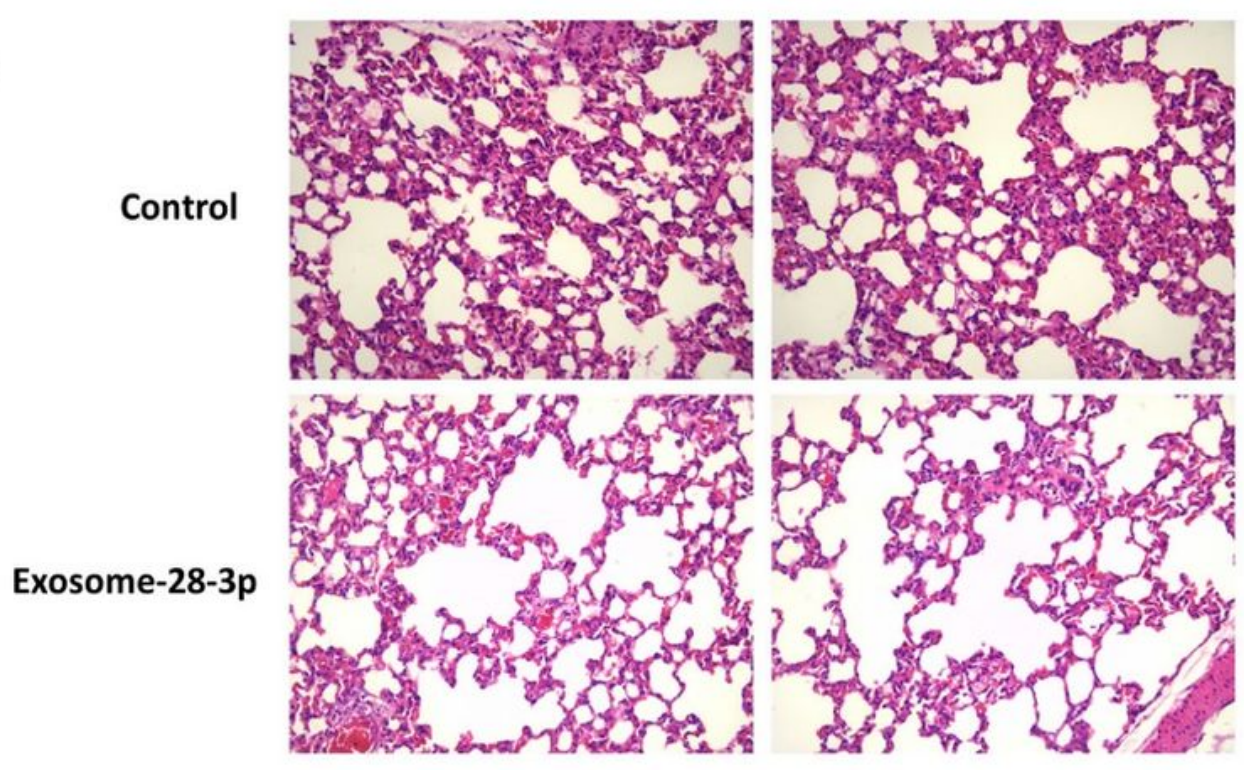

Figure 6 
Effect of miR-28-3p-modified exosomes on PECs apoptosis in vivo. (A) miR-28-3p expression in PECs of PE mouse model treated with miR-28-3p-modified exosome. (B) API5 protein expression in PECs of PE mouse model treated with miR-28-3p-modified exosome. (C). TUNEL assay for PECs of PE mouse model treated with miR-28-3p-modified exosome. (D) Representative hematoxylin and eosin staining images of $P E$ lung tissues. Values are means \pm SEM. $(*)$ denotes the difference between groups $(P<.05)$. 